\title{
Enfoque de la política fiscal en \\ Venezuela en el período 2000-2016
}

\section{Approach to fiscal policy of Venezuela for the period 2000-2016}

DOI: http://dx.doi.org/10.17981/econcuc.39.2.2018.06

Artículo de investigación. Fecha de recepción: 15/06/2018 Fecha de aceptación: 10/10/2018

Armando Urdaneta M.

Universidad Metropolitana del Ecuador (Ecuador) ajum69@gmail.com

\author{
María Elena Pérez Prieto \\ Corporación Universitaria del Caribe - CECAR (Colombia) \\ maria.perezpr@cecar.edu.co
}

Marilú Tibisay Acurero Luzardo

Corporación Universitaria del Caribe - CECAR (Colombia)

marilu.acurerol@cecar.edu.co

Para citar este artículo:

Urdaneta, A., Pérez, M. y Acurero, M. (2018). Enfoque de la política fiscal en Venezuela en el período 2000-2016. Económicas CUC, 39(2). 95-108. DOI: http://dx.doi.org/10.17981/econcuc.39.2.2018.06

\section{Resumen}

La investigación analiza el enfoque de la política fiscal en Venezuela para el período 20002016, determinando su efectividad en cuanto al manejo del déficit fiscal, base impositiva e ingreso fiscal, con base a datos publicados por la Oficina Nacional de Presupuesto - ONAPRE y el Banco Central de Venezuela (BCV, 2016). El estudio se basa en la teoría de la Curva de Laffer (1986). El enfoque metodológico es analítico y asume un diseño de investigación documental. Los resultados corroboran que el aumento de la base impositiva acrecienta el déficit fiscal, y disminuye el ingreso fiscal en términos reales..

Palabras clave: Déficit fiscal, base impositiva, ingreso fiscal, curva de Laffer

\section{Abstract}

The investigation analyzes the approach of the fiscal policy in Venezuela for the period 2000-2016, determining the effectiveness of the same in the management of the fiscal deficit, tax base and fiscal income, based on data published by ONAPRE (Oficina Nacional de Presupuesto) and the BCV (Banco Central de Venezuela, 2016). The study is based on the theory of the Laffer Curve (1986). The methodological approach is analytical and assumes a documentary research design. The results corroborate that the increase in the tax base increases the fiscal deficit and decreases the fiscal income in real terms.

Keywords: Fiscal deficit, tax base, fiscal income, Laffer curve 


\section{Introducción}

La curva de Laffer constituye uno de los métodos de análisis económico más conocido en el mundo económico, la misma fue diseñada por el economista Arthur Laffer, quien plantea que subir la tasa del impuesto no necesariamente aumenta la recaudación, porque la base tributaria decrece. Esta curva viene a ser una representación gráfica que muestra cómo afecta a la economía de un país el hecho de que los ingresos gubernamentales sean aumentados mediante impuestos y busca explicar de manera clara que no necesariamente la subida del impuesto o carga tributaria por parte del gobierno va a significar obtener más dinero con esa medida.

En este entendido, las autoras Chumaceiro y Hernández (2016), expresan que la "sociedad se somete a los intereses generales del Estado, en función de un orden jurídico interno, acuerdos y políticas públicas. De allí, las políticas públicas subordinan intereses e impulsan estrategias definidas como prioritarias en una determinada área de acción" (p. 106). Ahora bien, la política tributaria, según Chumaceiro (2013) "se inspira en el principio de que los impuestos deben ser distribuidos con arreglo a la capacidad de pago de cada ciudadano" (p. 42).

Así, tal como lo plantea Aliaga y Oropeza (2015), la carga tributaria se refiere al peso de los impuestos que son soportados por las personas naturales o jurídicas. Entonces, cuando esta carga es elevada, existe la tendencia a no pagar impuestos como también se observaría el no pago de impuestos para las personas discapacitadas, que según los autores Araque y Suárez (2017) exponen como, de acuerdo con la ONU, para el 2025 y hasta el 2050 serán 86 millones más de personas discapacitadas, lo que equivaldría al $22.6 \%$ de la población total mundial estimada para esa epóca.
De ello se desprende como a medida que se aumenta la alícuota impositiva, se va reduciendo la cantidad de dinero que las personas están dispuestas a tributar. Adicionalmente, otro factor que influye en el cumplimiento es el comportamiento natural de las personas hacia el riesgo. De allí que esta política fiscal debe ser evaluada a la luz del comportamiento de los agentes económicos receptores de la misma, para garantizar su eficacia como mecanismo de aumento del ingreso nacional.

Las políticas fiscales constituyen instrumentos de gestión pública, las cuales son utilizadas por los gobiernos para conducir y orientar la actividad económica de un país y lograr a través de éstas, el cumplimiento de objetivos que se propone el Estado, tales como generar crecimiento económico, aumento del Producto Interno Bruto (PIB), el incremento del Producto Interno Bruto per cápita y una distribución equitativa de la renta nacional, entre otros. De este modo, las políticas fiscales implementadas por los gobiernos en conjunto con otras políticas macroeconómicas, deben estar dirigidas a impactar favorablemente el bienestar social de la población, en términos de mejoramiento progresivo de la calidad de vida de la sociedad.

Por su parte Fernández, Rodríguez y Parejo (2008), describen la política fiscal como el conjunto de variaciones en los programas de gastos y de ingresos del gobierno, realizados con el fin de colaborar al logro de los objetivos de la política macroeconómica. Mediante estas variaciones se piensa que el o los gobiernos ejercen un poderoso impacto sobre la demanda agregada, y por consiguiente, dado un nivel de precios, sobre la producción y el empleo. En definitiva, la política fiscal constituye la vía más importante para mantener o mejorar el llamado estado del bienestar (caracterizado políticamente, como una actuación solidaria de la sociedad que trata de garantizar cierto nivel de vida a los ciudadanos, poniendo una red protectora que aminore desigualdades). 
Fernández, Rodríguez y Parejo (2008), ven en la política fiscal el instrumento mediante el cual el Estado realiza sus funciones de gasto público, impuestos, estabilización y planificación de la política económica de la nación. Por su parte, Maza y González (1992), plantean que el propósito de la política fiscal es darle mayor estabilidad al sistema económico, mientras consigue el objetivo de ocupación plena, manteniendo o buscando un punto de equilibrio entre gasto fiscal y el ingreso fiscal, en aras de mantener el balance macroeconómico sin grandes inclinaciones en el empleo ni en la actividad económica, y previniendo severos ataques de inflación a la vez que asegura una adecuada tasa de crecimiento económico mediante una apropiada distribución de la renta entre los diversos grupos de la economía, y una eficiente asignación de recursos en toda la economía.

En el caso venezolano, según cifras de la Oficina Nacional de PresupuestoONAPRE (BCV, 2015), a partir de 1989, la política fiscal ha sido de carácter expansiva, presentando un déficit fiscal estructural el cual ha crecido $307 \%$ en términos reales a lo largo del periodo sujeto a estudio, pasando del 5\% al $21 \%$ del Producto Interno Bruto Real (PIBR) al término de 2015, ello debido a que los ingresos fiscales por concepto de impuesto netos sobre los productos y los derechos de importación como componentes del PIBR en la oferta global de la economía solo han crecido $67 \%$ en términos reales, mientras el gasto al consumo final del gobierno como parte de la demanda agregada interna ha crecido $121 \%$, (BCV, 2015); lo que representa uno de los déficits más altos de Latinoamérica. Esto produjo un aumento de la base monetaria de 5.232.061.000 Bs. Fuertes, en el año 2000 hasta 1.542.338.037.000 Bs. Fuertes al cierre del año 2015, según reporte de los agregados monetarios por parte del BCV (2016), todo ello para financiar las dos terceras parte del déficit público y el restante ha sido por la vía del endeudamiento interno y externo de la nación.

Ese incremento de la masa monetaria sin la debida correspondencia con el aumento de la renta real y el nivel de reservas internacionales se ha traducido en un incremento general de los precios y con ello de la inflación, presentando un aumento promedio anual del 35\% (BCV, 2015). En tanto, el PIBR solo muestra un crecimiento promedio anual del 1,29\%, mientras que la tasa desempleo ha mostrado un comportamiento promedio del $10 \%$ en el período.

De allí, que el presente estudio de investigación tiene como objetivo analizar el enfoque de la política fiscal en Venezuela en el período 2000-2016, para determinar la efectividad de la misma en cuanto al manejo del déficit fiscal, base impositiva e ingreso fiscal, partiendo de la hipótesis que el incremento de la base impositiva en el lapso sujeto a estudio ha acrecentado el déficit fiscal, debido a la disminución del ingreso fiscal en términos reales. En la primera parte, se presenta la situación estudiada y el objetivo general. Posteriormente, se expone la fundamentación teórica y metodológica de estudio. Así como la discusión y análisis de los resultados, para finalizar con las conclusiones obtenidas.

\section{Desarrollo}

Para iniciar la discusión teórica de la situación estudiada es necesario establecer un contraste entre las posturas Keynesianas y Poskeynesianas desarrolladas por John Maynard Keynes durante la década de los años treinta, como consecuencia de la gran depresión de la economía norteamericana; la cual según Ekelund (2003), en uno de sus postulados fundamentales de su obra "La Teoría General" hace énfasis en el uso de la política fiscal por parte de los gobiernos para complementar los mecanismos de mercado del sector privado, en aras de resolver los problemas de desempleo existentes. 
Keynes creía que probablemente el sector privado no haría las inversiones necesarias para aumentar la tasa de empleo, razón por la cual según Ekelund (2003), sugería que los gastos e impuestos del gobierno actuaran como elementos compensadores a fin de aliviar el desempleo y la subproducción, oponiéndose en forma enérgica al uso del recurso exclusivo de la política monetaria para estabilizar la economía. Es por ello, que Keynes pensaba que la inversión agregada privada estaba condicionada en gran manera por las expectativas sobre precios y beneficios, donde una disminución rápida del nivel de precios puede prácticamente reducir el nivel de inversión, incluso en el caso de los tipos de interés decrecientes. Las quiebras y condiciones adversas de los negocios seguirían casi con toda certeza a la disminución de los precios (Ekelund, 2003).

En ese sentido, expresa el mismo autor como Keynes argumentaba sobre la base de sus desarrollos teóricos que el gobierno debía usar sus poderes para gravar impuestos y para gastar, con el fin de influir en el ciclo económico. El gasto gubernamental debía representar una inyección directa de inversión pública en el flujo de la renta, el mismo podía financiarse mediante impuestos progresivos, lo que reducía el consumo, pero en menor cantidad que la correspondiente al impuesto. Por ello, proponía un programa planificado en gran escala de política fiscal discrecional, así como un fortalecimiento de los estabilizadores incorporados como impuestos progresivos. En pocas palabras, el gobierno tenía que estar preparado para proporcionar las condiciones de pleno empleo.

En contraposición a los planteamiento anteriores, para Fernández, Rodríguez y Parejo (2008) se encuentra la corriente monetarista encabezada por el economista Milton Friedman, premio nobel de economía 1976, quien platean que la manipulación de las variables nominales no tienen un efecto sostenible sobre las variables reales a mediano y largo plazo, partiendo de la premisa del valor neutral del dinero desarrollado por David Hume en 1776; razón por la cual un aumento del gasto público para estimular el crecimiento económico en los ciclos recesivos como lo planteaba Keynes, solo traería consigo un incremento general de precios en el mediano y largo plazo y si bien en corto plazo como lo plantea Friedman se producía un leve crecimiento económico y una disminución del desempleo en el mediano y largo plazo, estos retornarían a la posición inicial donde comenzó la utilización de políticas fiscales y monetarias expansivas (Ekelund, 2003).

Para Friedman, según lo expuesto por Ekelund (2003), la economía es básicamente constante y autorregulada, si el papel del gobierno y el banco de reserva federal consisten en proporcionar un entorno predecible y estable en el que los procesos económicos pueden funcionar sin trabas, con mayor eficiencia a fin de maximizar el bienestar económico. Así pues, la mínima intervención del gobierno, los presupuestos equilibrados, desregulación de los precios y una norma de crecimiento monetario forman parte de las premisas de los monetaristas.

En medio de estos planteamientos, surge la tesis sobre la Curva de Laffer, la cual tiene origen en las contribuciones de Arthur Laffer; quien como él mismo menciona, es un concepto que se remonta al siglo XIV. Ya Keynes había planteado la misma teoría como señala Bour (2009), y también lo menciona el padre de la economía, Adam Smith en su libro "The Wealth of Nations" (libro 5, Cap. II), citado por Aliaga y Oropeza (2015). Para ilustrar sus ideas, Laffer dibujó en una servilleta una curva en la que ilustraba el intercambio entre las recaudaciones y la tasa de impuesto (Wanniski, 1978). Este gráfico luego se denominaría Curva de Laffer (Figura 1). 


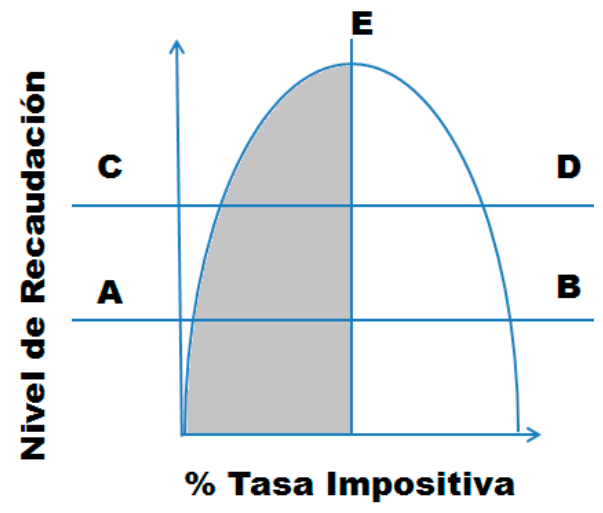

Figura 1. Curva de Laffer.

Fuente: Elaboración propia.

En términos generales, la Curva de $L a$ ffer (1986), según "The Ellipse: An Explication of the Laffer Curve in a Two-Factor Model», es la relación entre el nivel de impuestos y el nivel de recaudación impositiva (ingresos fiscales) para una economía. Si los impuestos son nulos, está claro que el nivel de ingresos públicos es también cero. Haciendo un análisis de las diversas posibilidades que propone la Curva de Laffer, se destaca lo siguiente: en el punto A, una pequeña tasa tributaria también haría aumentar las recaudaciones y aumentaría la producción. En el punto B, luego de imponer un impuesto, la producción superaría la economía del trueque, pero a través de un impuesto alto, la producción disminuiría y se podría tener menores recaudaciones. En los puntos C y D, un aumento de la producción o una reducción de los impuestos aumentarían los ingresos fiscales.

Lo mismo ocurriría en el caso extremo en el que el tipo impositivo fuese del $100 \%$, ya que en este caso el nivel de actividad sería cero. Por tanto, la curva de Laffer tiene un tramo creciente y un tramo decreciente. Aunque generalmente se atribuye este razonamiento a Laffer, y de ahí su nombre, lo cierto es que este resultado económico es muy antiguo, quizás por lo intuitivo del mismo.

Según Wanniski (1978), se presentan dos aspectos: a. Si el impuesto fuera igual al $100 \%$ del ingreso, la producción no existiría, debido a que toda ganancia que se obtendría de la producción la llevaría el Estado.

b. En el otro extremo, si el impuesto fuera cero, claramente los ingresos del Estado serían cero, no existiría ninguna obstrucción por parte del Estado. En esta economía, la productividad se maximizaría y la producción sólo se limitaría si los trabajadores realizaran un trade-off entre trabajo y ocio.

Por ello, ManKiw (2007) expone la Curva de Laffer para mostrar la relación dual existente entre la tasa de impuestos y la recaudación, esta relación entre las variables mencionadas genera dos efectos: renta y sustitución. El efecto renta como consecuencia del cambio en la capacidad adquisitiva de los contribuyentes, y el efecto sustitución refleja los cambios en las decisiones que toman los agentes económicos como resultado de la variación de impuestos.

Eso se puede notar muy claramente al tener una tasa de impuestos muy alta, la cual incentivaría a la evasión y mayor elusión de impuestos, o si se tuviera una tasa demasiado baja, donde entonces el gobierno no contaría con los recursos necesarios para poder cumplir con sus funciones de estado. Además, una medida de alza de impuestos generaría una reducción en el ingreso disponible, que se reflejaría en la disminución de la demanda agregada y un posterior decrecimiento en el producto. Por ello, es importante conocer cuál sería la tasa óptima, y si la hubiera, saber si es que nuestras autoridades están pendientes de ello.

\section{La paradoja de la economía venezolana}

En el caso venezolano, al igual que en la mayoría de los países de América Latina, según Manzano, Pineda y Ríos (2008), los gobiernos se han caracterizado por utilizar medidas de expansión fiscal y monetaria en aras de estimular el crecimiento económico, 
pero con frecuencia fracasan en su propósito de mantener la inflación dentro de los límites de moderación y control. Esto se debe a que la expansión del gasto público supera los ingresos fiscales, generándose constantes y agudos déficits fiscales, los cuales son financiados mediante el endeudamiento interno y externo, pero en la mayoría de los casos mediante expansión de la masa monetaria, la cual al crecer muy por encima de la renta real de la economía produce un desequilibrio entre el mercado de bienes y el mercado de dinero.

Según el economista venezolano Maza (2010), la economía venezolana ofrece un campo de investigación y análisis con rasgos de originalidad, lo que puede servir para explicaciones teóricas dentro del marco de la economía latinoamericana, que es de por sí un campo especial de estudio. La particularidad de este país, es en efecto, una economía en la cual la extraordinaria afluencia de los recursos procedentes de la explotación de un recurso natural no renovable como el petróleo; sin embargo, no se ha manifestado un crecimiento sostenido, sino en una tendencia a la recesión. Paralelamente, el fenómeno de la inflación es persistente, de difícil abatimiento en las condiciones actuales, mientras que en otros países de la región se ha logrado dominarla.

Venezuela, siendo potencialmente un país de oportunidades para la inversión real, es sustancialmente insuficiente, en tanto que el ahorro nacional se exporta, debido a una balanza de pagos desfavorables, la caída del producto interno bruto, el incremento de la demanda agregada y las importaciones, el alto costo del pago de la deuda externa, la fuga de capitales producto del sistema político existente, entre otras debilidades. Todo ello a pesar de los cuantiosos ingresos en dólares obtenidos por la República en los últimos 15 años por concepto de las exportaciones petroleras, acompañadas, con un exponencial creci- miento de los precios del petróleo, aunque con una disminución progresiva de los volúmenes de producción de petróleo a partir del año 2002 y con ello de las exportaciones de este rubro.

Otras exportaciones como las no petroleras, declinan ostensiblemente por falta de políticas de Estado eficientes, en materia de comercio exterior y encarecimiento de los costos, todo esto debido a la política de control de cambio instaurada a partir de año 2002 por el gobierno venezolano. La importación de bienes y servicios en lugar de ser complementaria, es sustitutiva de la producción interna, porque ésta sufre de decaimiento por motivos de inseguridad jurídica, mediante la confiscación y expropiación de la propiedad privada, falta de apoyo social y expectativas económicas oficiales inciertas. Por ello, la dependencia del suministro externo mediante importaciones, para producir escasamente y para subsistir, es cada vez mayor, lo que imprime a la economía un sello de vulnerabilidad e inestabilidad acentuada.

En igual nivel de menoscabo se encuentra el sector de bienes agrícolas y agroindustriales, en cuanto a la inflación y escasez de éstos, debido a que el gobierno subsidia buena parte del consumo popular de alimentos mediante importaciones, propiciando la fuga o erogación de divisas extranjeras. Esto indudablemente afecta la capacidad de ahorro de la nación pues utiliza, incluso, sus propios agentes de distribución y comercialización, e incrementa con ello el costo del subsidio y dilata el aparato burocrático, en lugar de sustituir esos subsidios en moneda local o promover e incentivar la producción nacional de alimentos. El Estado se ha apropiado de grandes extensiones de tierra laborable, con el pretexto de su ociosidad, pero posteriormente no son puestas a producir, de tal manera que el latifundio privado se convierte en un latifundio social; al mismo tiempo es notable la ausencia de un plan agrícola que 
permita orientar la recuperación del potencial agrícola.

Por otra parte, con la caída de la producción interna, el modo y los medios de financiamiento, así como de la salida de capital al exterior han sido objeto de estudio, debido al régimen de control de cambio integral, implementado como política cambiaría del gobierno a partir del año 2003. Los indicadores señalan que la salida de fondos no concuerda con las restricciones y condicionamientos del control cambiario, menos aun cuando el mercado permuta de divisas ha sido incorporado al Banco Central de Venezuela, cuando hasta el año 2008 había sido manejado por Petróleos de Venezuela (PDVSA).

Por otra parte, la cantidad de dinero adjudicado al exterior, representa en divisas tres veces más de las que recibe el $\mathrm{BCV}$, hecho que constituye una contrariedad en tanto que escasean las divisas para las importaciones necesarias. La balanza de pagos padece esta debilidad en su cuenta de capital y financiamiento, ubicándose en un mayor estatus la tendencia a la reducción de los saldos activos de la balanza comercial y el nivel decreciente de las reservas monetarias internacionales. Es paradójico, luego de haber ingresado al tesoro nacional entre los años 2000 y 2015, según cifras del BCV, por la vía de las exportaciones de bienes, servicios y endeudamiento, más de 1.350.000.000.oo de dólares, el triple de los ingresos percibidos en las cuatro décadas anteriores, Venezuela sea la única economía de América Latina con una inflación en tres dígitos.

De acuerdo con las proyecciones del Instituto Nacional de Estadísticas (INE), la población venezolana ha crecido por encima de los 28 millones de habitantes, pero su calidad de vida se ha deteriorado debido a que el salario mínimo desde el año 2003 no cubre la canasta básica de consumo y, desde el año 2008, ni siquiera cubre la canasta básica de consumo de alimentos; el alojamiento es precario, con un déficit de vivienda que supera el millón de viviendas, los servicios públicos muestran síntomas de deterioro de calidad en el servicio, y el desempleo efectivo afecta a más de un tercio de la población activa. El gobierno confronta una carestía evidente de recursos fiscales ordinarios debido, según el BCV (2016), a que el ingreso fiscal en promedio en los últimos 17 años, es $45 \%$ inferior al gasto público, lo que constituye un déficit fiscal promedio para el mismo periodo de $14 \%$ del PIB real, en tanto su requerimiento de gasto aumenta y con ello el desplazamiento de la inversión privada como motor dinamizador de la economía.

Análisis de la política fiscal en Venezuela en el periodo 2000-2016

Venezuela en los últimos 17 años se ha caracterizado por manejar una política fiscal expansiva, donde el gasto público en términos promedio supera en un $45 \%$ al ingreso fiscal por periodo según cifras de la ONAPRE, estimulando con ello la demanda agregada interna, la cual creció en un $68 \%$, mientras que el PIBR solo aumentó $38 \%$, según el sistema de cuentas nacionales del Banco Central de Venezuela (BCV, 2016).

Es importante resaltar, como ese tipo de políticas, se aplican especialmente cuando una economía está en recesión y necesita un impulso para expandirse, pero como resultado se tiende al déficit fiscal, como es el caso venezolano, el cual presenta un nivel promedio 14,01\% del PIBR. Esto como se ha señalado en párrafos anteriores, ha traído como resultado para este período, el desencadenamiento de un espiral inflacionario al que ha sido sometida la sociedad venezolana con una tasa acumulada de más del $6728 \%$ y un nivel de endeudamiento público total del Estado que ha crecido más $204 \%$ entre los años 2000 y 2015, según cifras del BCV (2016). 
Ante tal situación, Manzano, Pineda y Ríos (2008), revelan una caída del poder adquisitivo de los venezolanos y por ende un aumento de la pobreza, todo ello también impulsado por un incremento de las importaciones de bienes y servicios, medidas a precios constantes, las cuales crecieron en un $116 \%$ para el período, según cifras del BCV (2016). Mientras que las exportaciones de bienes y servicios, medidas a precios constantes, decrecieron en un $61 \%$. Esto convierte a Venezuela en un país con una economía de puerto altamente dependiente de otras economías, donde cada vez más se importan bienes y servicios para satisfacer su demanda agregada interna, por ende, la tasa de inflación foránea sumada a la tasa de inflación nacional, es traslada al consumidor final, mermando cada vez más su poder adquisitivo.

Por otra parte, otro de los aspectos importantes a señalar en este crecimiento exponencial de las importaciones, es como este ha contribuido en gran medida al déficit fiscal que presenta el Estado; además de haber incrementado la deuda pública externa, produciendo con ello un detrimento considerable en la capacidad de ahorro de la nación, como otra forma para cubrir el déficit, en el que a pesar de haber ingresado al país más de 950.000 millones de dólares según cifras del BCV (2015) por concepto de exportaciones, en los últimos 17 años; el ahorro de la nación decreció de 20.471 millones de dólares en el 2000, a 16.370 millones de dólares en el 2015, es decir, se produjo una descapitalización de las reservas internacionales del país de más del $20 \%$.

Adicionalmente, desde el año 2004 la mayor parte de los ingresos en divisas extranjeras, no son entregadas por PDVSA al BCV, sino que son manejadas a discrecionalidad del gobierno, mientras que las reservas internacionales óptimas son consideradas para la República, en el orden de los 30.000 millones de dólares y el excedente por concepto de reservas excedentarias, son transferidas al Fondo de Desarrollo Nacional(FONDEN) y otros fondos paralelos no fiscalizables por el poder legislativo.

En ese mismo orden de ideas, Urdaneta (2015) afirma que el comportamiento trimestral de las variables reales en el manejo de la política fiscal, tales como el gasto de consumo final del gobierno y los impuestos netos sobre los productos del gobierno, no tienen un alto impacto en el fenómeno inflacionario en Venezuela, y que el mismo obedece a otros elementos de la política económica, tales como el incremento de la masa monetaria, para financiar el estructural déficit fiscal que se puede evidenciar en términos reales; el cual pasó de $5 \%$ del PIBR en 1997 a $10 \%$ del PIBR en 2013, con un crecimiento en términos reales en el periodo sujeto a estudio de $307 \%$, producto de un aumento del $196,87 \%$ del gasto de consumo final del gobierno, mientras los impuestos netos sobre los productos del gobierno solo se incrementaron $111,21 \%$.

Igualmente, Guerra (2008), señala que la función impositiva del Estado venezolano se materializa básicamente en la aplicación de los impuestos al valor agregado (IVA) e impuesto sobre la renta (ISLR), los cuales representan más del $80 \%$ de la recaudación de los ingresos del Gobierno Nacional.

El total de ingresos y fuentes de financiamiento presentó un incremento del $242 \%$ para el periodo $2005-2009$ y de $330 \%$ para el periodo 2009-2014, siendo esto significativo por cuanto se está financiando el gasto del gobierno cada vez más por la vía del endeudamiento. De la revisión de los saldos, en la ejecución presupuestaria se aprecia como el año 2005 y la serie de años del 2009 a 2014 reflejan saldos negativos o déficit con lo cual se evidencia un manejo exagerado 
del criterio Keynesiano, en cuanto a la intervención del Estado en materia de política fiscal, esto por cuanto el incremento del déficit para el periodo 2005-2009 fue de $166 \%$, mientras que para el periodo 2009-2014 alcanzó un $1.819 \%$, once (11) veces superior al déficit del periodo 2005-2009.

El desempeño del gobierno en materia de política fiscal refleja el incumplimiento de todos los criterios establecidos en la literatura económica financiera sobre el manejo de la política fiscal, sobre todo cuando era evidente como la economía venezolana se convertía en una economía de puerto, dado que cada vez se hacía más dependiente de las importaciones de bienes para el consumo y un aparato productivo cada vez más destruido, sin aparente posibilidad de reconstrucción. Es necesario, un cambio en el manejo de la política fiscal en Venezuela, que incentive el pleno empleo y elimine las restricciones al desenvolvimiento normal de la economía.

De igual modo, Urdaneta (2015) señala como luego de haber analizado detalladamente en forma retrospectiva la dimensión del sector público, se puede resumir que al cierre del año 2014, la mayor parte de las fuentes de financiamiento del presupuesto público del país recaen sobre el sector no petrolero, con el $58 \%$, a pesar de ser una economía dependiente de la renta petrolera, la cual hoy apenas representa $20,37 \%$ de las fuentes de financiamiento, mientras el $21,63 \%$ restante se financia por la vía del endeudamiento. Es un error pretender estimular la actividad económica a través de medidas monetarias y fiscales con miras a reducir el desempleo, lo cual resulta exponerse a desatar un proceso inflacionario sin conseguir el objetivo perseguido, como ha ocurrido en el caso venezolano, donde el financiamiento monetario del 43,73\% déficit público en los últimos 15 años se ha traducido en una inflación acumulada del 2338,57\% según cifras del índice nacional de precios al consumidor reportado por el Banco Central de Venezuela (BCV, 2015).

Ante los planteamientos anteriores, se analizarán los datos estadísticos de los indicadores fiscales durante el período de estudio, tomando como técnica la Curva de Laffer para corroborar o no la hipótesis planteada.

\section{Metodología}

Dentro del marco procedimental que plantea el objetivo general de investigación propuesto, así como el nivel de profundidad, el estudio se tipifica como analítico, al ser un estudio enfocado en los efectos de la política fiscal en la economía venezolana en un periodo determinado, considerando como referentes de análisis los elementos teóricos contenidos en el efecto Laffer en su enfoque fiscal. Según Hernández, Fernández y Baptista (2008), la investigación analítica permite ir más allá que la mera descripción, lo que implica contrastaciones de teorías e interpretación de resultados, permitiendo hacer una reflexión de lo revisado.

Su diseño es de carácter documental, la fuente de información es secundaria, compuesta por los datos estadísticos de los indicadores de políticas fiscal en el periodo 2000-2016 emanados por la Oficina Nacional de Presupuesto y del Banco Central de Venezuela, órganos oficiales de información del área económica y fiscal en el país. Hernández, Fernández y Baptista (2008) definen el diseño documental como la revisión sistemática, rigurosa y profunda del material documental de cualquier clase. Se procura el análisis de los fenómenos o el establecimiento de la relación entre dos o más variables y al optar por este tipo de estudio, el investigador utiliza documentos, los recolecta, selecciona, analiza y presenta resultados coherentes 


\section{Resultados}

$\mathrm{Al}$ analizar la curva de Laffer aplicada a la política fiscal en Venezuela en los últimos 16 años como se puede observar en la figura 2 , tomando como base impositiva la diferencia porcentual promedio en el periodo del Índice Nacional de Precios al Consumidor (INPC), con respecto al aumento de la unidad tributaria, asumiendo como año base 1997, con base 100 puntos, se observa que ya para el año 2000 el INPC había crecido 44,35\% más que la unidad tributaria y así sucesivamente hasta el año 2016, lo cual implica un aumento de la base impositiva para lograr una mayor recaudación fiscal.

En la figura 2, también se detalla que durante el período de estudio el déficit fiscal recurrente que padece la economía venezolana desde el año 1989 se mantuvo, pero a partir de que la base impositiva aumento el mismo subió a 588,71 puntos. En el año 2012, este pasa a ser mayor que el ingreso fiscal en términos reales, como puede evidenciarse en el período que la ba- se impositiva varío de 144,35 a 336,79. Los ingresos fiscales crecieron, pero el gasto fiscal también, aunque en menor medida, por lo que hubo una reducción del déficit fiscal en el periodo 2000-2008. Sin embargo a inicio del año 2009, comienza a propiciarse un efecto inverso por la caída de los precios del petróleo, con aumento de la inflación y ampliación de la base impositiva en mayor cuantía por parte del gobierno, buscando cubrir la brecha de ingresos fiscales dejados de percibir por la actividad petrolera; lo cual se tradujo en una caída de los ingresos fiscales muy por debajo del gastos fiscal en términos reales y con un aumento aun mayor del déficit fiscal, el cual pasó en valores promedios del $13 \%$ con respecto al PIBR en el lapso 2000-2008 a 17\% en el lustro 2009-2016.

Esta situación, trajo como consecuencia a partir del año 2013, como se observa en la figura 3, la elasticidad ingreso fiscal real-base impositiva pase a ser inelástica lo que permite que un aumento de la base impositiva tenga un efecto insignificante en el aumento del ingreso fiscal, el cual

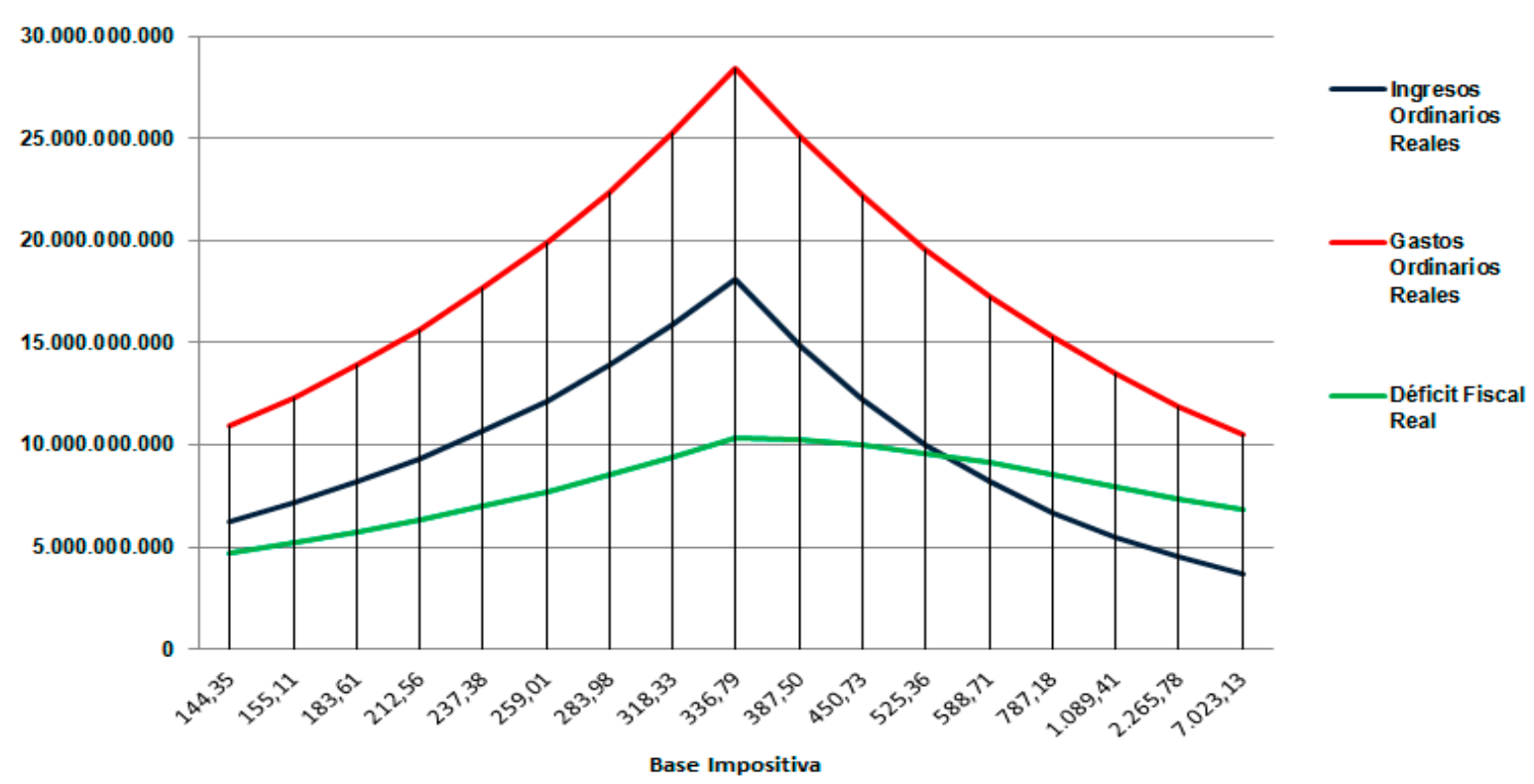

Figura 2. Comportamiento de los indicadores de política fiscal en función de la evolución de la base impositiva.

Fuente: Elaboración propia en base a datos de la ONAPRE y BCV (2016). 


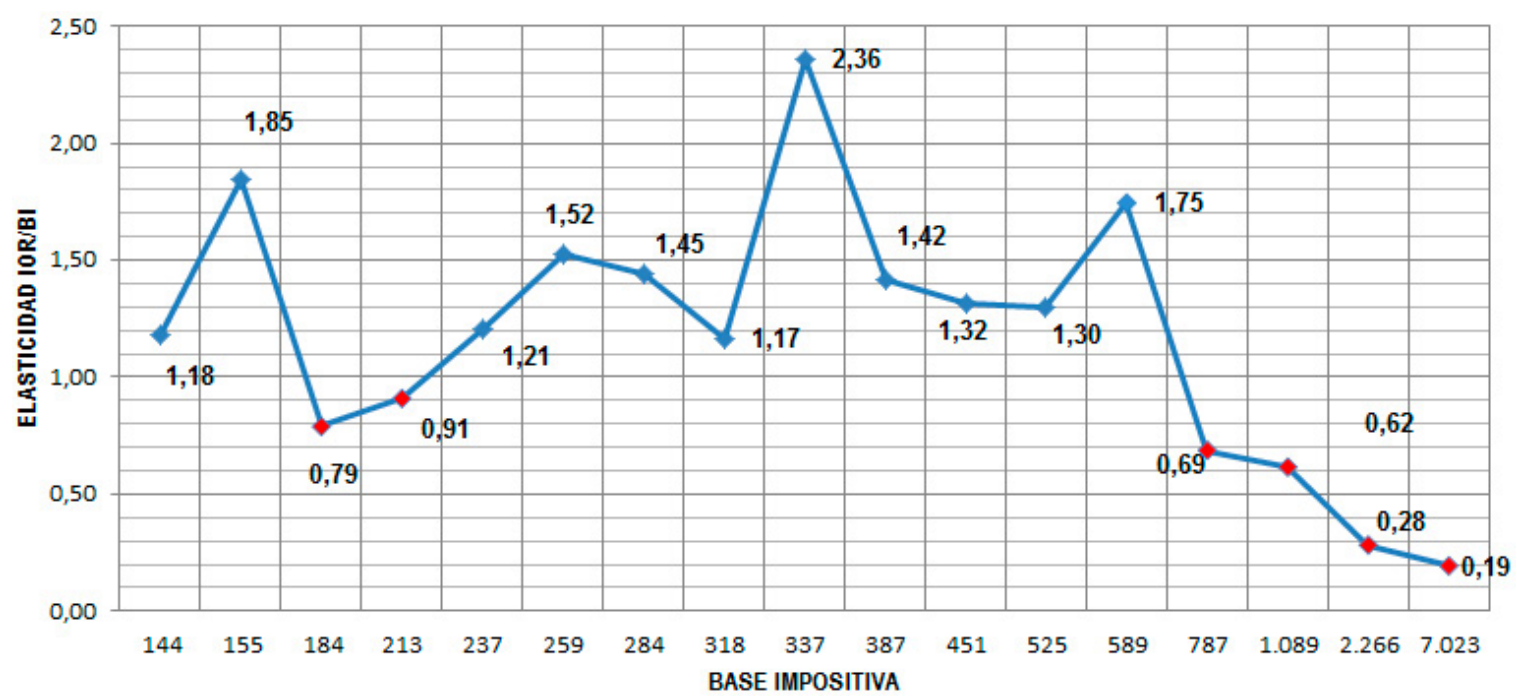

Figura 3. Comportamiento de la elasticidad ingreso fiscal-base impositiva en función de la evolución de la base impositiva.

Fuente: Elaboración Propia en base a datos de la ONAPRE y BCV (2016).

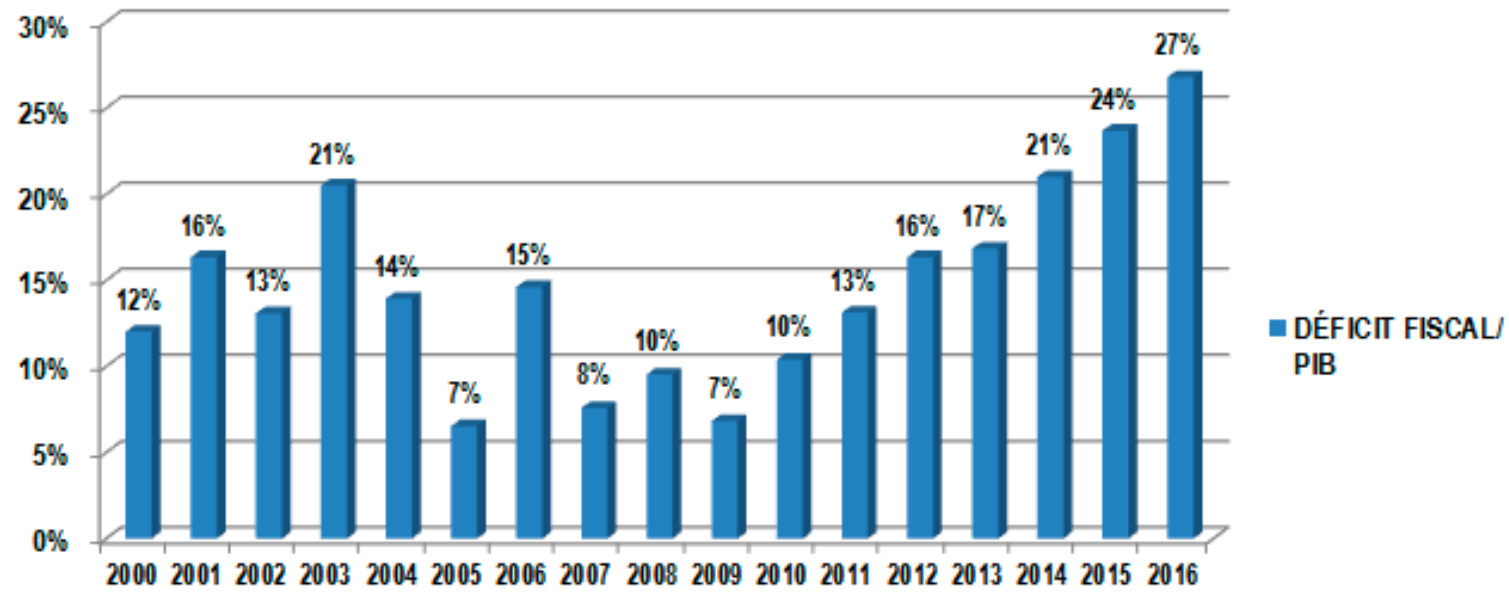

Figura 4. Comportamiento déficit fiscal/PIB en el período 2000-2016.

Fuente: Elaboración propia en base a datos de la ONAPRE y BCV (2016).

está por debajo de la tasa inflación que se genera, como consecuencia de la baja producción de las empresas, debido al incremento de la carga impositiva que el Estado hace sobre ellas. Esto trajo como consecuencia una disminución en los niveles de inversión y producción, porque tienen que destinar mayores recursos financieros para el pago de impuestos.
En la figura 3, se evidencia que el valor más alto de elasticidad en el período sujeto a estudio fue 2,36 en el año 2008, es decir un aumento de la base impositiva en $1 \%$ se traducía en un incremento del 2,36\% del ingreso fiscal en términos reales. Durante los años 2002-2003 y 2013-2016, se presenta el fenómeno de inelasticidad, es decir el enfoque de la política fiscal por el lado 
del ingreso ha sido errado, aumentando la base impositiva, en lugar de disminuir el gasto fiscal en términos reales; en el resto de datos anuales analizados se presenta un componente de elasticidad, es decir la variación de la base impositiva tuvo un efecto mayor en la variación del ingreso fiscal en términos reales. La perspectiva de la política fiscal por el lado del ingreso ha sido aceptable acrecentando la base impositiva.

Seguidamente, puede observarse en la figura 4, el comportamiento del déficit fiscal con respecto al PIBR. Como los años, en los cuales el coeficiente de elasticidad mostrado en la figura 3 , es inferior a 1 es decir hay un componente inelasticidad, el déficit fiscal muestra una evolución creciente de manera permanente, sobre todo en el período 2009-2016, en lugar de presentar un comportamiento oscilante como en el periodo 2000-2008.

Ahora bien, el escenario antes planteado es producto del ajuste inadecuado del valor de la unidad tributaria con respecto al aumento del INPC, como se muestra en la Tabla 1, entre el valor ideal de la unidad tributaria y el valor estimado por el ejecutivo nacional, así como el incremento anual de la base impositiva. En el período 2000-2009 no se observan diferencias significativas entre el valor ideal de la unidad tributaria y el valor real estimado por el gobierno. Sin embargo, partir el año 2010 comienza a producirse discrepancias prominentes; puesto que el déficit fiscal ha mantenido un incremento recurrente con respecto al Producto Interno Bruto Real, traduciéndose en un crecimiento de este por encima de los ingresos fiscales a partir del año 2012, como se observa en la figura 2. Así mismo, es importante señalar en la Tabla 1, los años 2001 y 2008, donde hubo una reducción de la base impositiva, al ser estos los años donde la elasticidad ingreso fiscal-base impositiva fue más elástica, es decir dicha reducción significo mayores ingresos fiscales.
TABLA 1.

Comportamiento del valor ideal y real de la unidad tributaria en el período 2000-2016.

\begin{tabular}{|c|c|c|c|}
\hline & $\begin{array}{l}\text { Valor Ideal } \\
\text { de la Unidad } \\
\text { Tributaria }\end{array}$ & $\begin{array}{c}\text { Valor Anual } \\
\text { de la Unidad } \\
\text { Tributaria }\end{array}$ & $\begin{array}{l}\text { Evolución del } \\
\text { Incremento de la } \\
\text { Base Impositiva }\end{array}$ \\
\hline 2000 & Bs. 11,60 & Bs. 11,60 & $0,00 \%$ \\
\hline 2001 & Bs. 13,04 & Bs. 13,20 & $-1,20 \%$ \\
\hline 2002 & Bs. 17,04 & Bs. 14,80 & $15,10 \%$ \\
\hline 2003 & Bs. 21,51 & Bs. 19,40 & $10,90 \%$ \\
\hline 2004 & Bs. 25,70 & Bs. 24,70 & $4,05 \%$ \\
\hline 2005 & Bs. 29,61 & Bs. 29,40 & $0,70 \%$ \\
\hline 2006 & Bs. 34,36 & Bs. 33,60 & $2,27 \%$ \\
\hline 2007 & Bs. 41,29 & Bs. 37,63 & $9,73 \%$ \\
\hline 2008 & Bs. 45,28 & Bs. 46,00 & $-1,56 \%$ \\
\hline 2009 & Bs. 56,65 & Bs. 55,00 & $3,00 \%$ \\
\hline 2010 & Bs. 72,06 & Bs. 65,00 & $10,86 \%$ \\
\hline 2011 & Bs. 91,94 & Bs. 76,00 & $20,98 \%$ \\
\hline 2012 & Bs. 110,43 & Bs. 90,00 & $22,69 \%$ \\
\hline 2013 & Bs. 172,48 & Bs. 107,00 & $61,20 \%$ \\
\hline 2014 & Bs. 282,87 & Bs. 127,00 & $122,74 \%$ \\
\hline 2015 & Bs. 509,17 & Bs. 150,00 & $239,45 \%$ \\
\hline 2016 & Bs. $1.833,02$ & Bs. 177,00 & $935,61 \%$ \\
\hline
\end{tabular}

Fuente: Elaboración propia a partir de datos de ONAPRE y BCV (2016).

\section{Conclusiones}

Primeramente, se corrobora la hipótesis planteada por el presente estudio, es decir, el incremento de la base impositiva en el lapso sujeto a estudio ha acrecentado el déficit fiscal, debido a la disminución del ingreso fiscal en términos reales. A partir del año 2012, año en el que se comenzó a elevar el valor del impuesto, también acrecentó el déficit fiscal, generando esto una disminución del ingreso fiscal en términos reales. Esto se debe, a que parte de los recursos que las empresas pudiesen dedicar a aumentar la producción para pagar mayores contribuciones fiscales 
de manera sustentable y sostenible en el mediano y largo plazo, deben dedicarlos para el pago de impuestos, debido a la creciente carga impositiva que el Estado les impone, cuestión que las termina haciendo improductivas (disminuyendo sus niveles de producción y productividad) y por ende contribuyen en menor medida al fisco en términos reales.

Así mismo, esta disminución de la producción, trae como consecuencia un problema de oferta interna en la economía y con ello una inflación de demanda que termina acrecentando exponencialmente los precios muy por encima del crecimiento de la renta real, dilapidando el ingreso fiscal en términos reales y amplía el tamaño del déficit fiscal en el caso venezolano.

Por ello, se hace necesario de manera impretermitible ajustar la unidad tributaria al valor ideal señalado en la Tabla 1 para crear incentivos fiscales y poder lograr aumentar la producción nacional, siempre tomando en cuenta el coeficiente de elasticidad ingreso fiscal en términos reales y aumento de la base impositiva, como herramienta para medir los efectos de la política fiscal.

Igualmente, urge reestructurar el gasto fiscal en cuanto a su dimensión y calidad, para hacerlo más eficiente, que propenda a disminuir el peso del déficit fiscal sobre el producto interno bruto real, mediante el aumento del PIBR y disminución del gasto público corriente, para bajar la inflación que se produce por la monetización del mismo y la escasez de oferta interna de bienes y servicios. En pocas palabras, enfocar la política fiscal por el lado del gasto y no del ingreso.

Esta política de expansión del consumo, a través del aumento del gasto público ha generado altos niveles inflacionarios, aunado al aumento de la carga impositiva creando un espiral negativo que atenta contra el crecimiento empresarial y el aumento de la oferta instantánea.

\section{Referencias}

Aliaga, J. y Oropeza, A. (2015). Análisis experimental de la curva de Laffer y la evasión fiscal en Bolivia. Revista LAJED. (24). 121-153.

Araque, F. y Suárez, O. (2017). Equidad Ética-Jurídica de la Ciencia, para la Emancipación del Conocimiento y los Saberes. Juridicas CUC, 13(1). 97-120. http://dx.doi.org/10.17981/juridcuc.13.1.2017.5

BCV. (2016). Información Estadísitca. Indicadores. [Online]. Recuperado de http://200.74.197.130/c2/indicadores.asp

BCV. (2015). ABC Económico. [Online]. Recuperado de http://200.74.197.130/c1/ abceconomico.asp

Bour, E. (2009). La teoría de "Public Choi$c e^{\prime \prime}$. [Working Paper]. Recuperado de http://ebour.com.ar/derecho/12-Public\%20Choice.pdf

Chumaceiro, A. (2013). Políticas internacionales, nacionales y políticas públicas tributarias en Venezuela como promotoras de la responsabilidad social de las empresas. [Tesis doctoradal]. Universidad Privada Dr. Rafael Belloso Chacín. Venezuela.

Chumaceiro, A. y Hernández, J. (2016). Países generadores de políticas públicas para el fomento de la cultura empresarial dirigida hacia la responsabilidad social. Revista Telos, 18(1). 103-120. Disponible en http://ojs.urbe.edu/index. php/telos/article/view/790

Ekelund, R. (2003). Historia de la teoría económica y de su método. Madrid: McGraw-Hill Interamericana.

Fernández, A. Rodríguez, L. y Parejo J. (2008). Política Económica. México, D.F.: McGraw-Hill.

Guerra, J. (2008). La inflación en Venezuela: propuesta para su enfrentamiento. Caracas: Instituto Latinoamericano de Ciencias Sociales. 
Hernández, R., Fernández, C. y Baptista, P. (2008). Metodología de la Investigación. México, D.F.: McGraw-Hill.

ManKiw, G. (2007). Principios de Economía. Madrid: Thomson.

Manzano, O., Pineda, J. y Rios G. (2008). Macroeconomía y petróleo. México, D.F.: Pearson. Recuperado de https:// www.researchgate.net/publica-

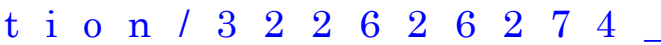
MACROECONOMIA_Y_PETROLEO_Con_Osmel_Manzano_Jose Pineda_y_German_Rios_Pearson_ Educacion_Mexico_2008.

Maza, D. y González, A. (1992). Tratado Moderno de Economía. Caracas: Panapo.

Maza, D. (2010). Visión Panorámica de la Economía Venezolana Actual. Revista Zeta. 1767. 18-21.

Urdaneta, A. (2015). Impacto de la política fiscal en Venezuela sobre el fenómeno de la inflación durante 1997-2013. Revista Desarrollo Gerencial. Recuperado de https://www.researchgate.net/ publication/282584194_IMPACT_OF_ FISCAL_POLICY_IN_VENEZUELA_ON_THE_PHENOMENON_OF_ INFLATION_DURING_1997-2013.

Wanniski, J. (1978). Taxes, Revenues and the LafferCurve. (The Wall Street Journal).
Armando Urdaneta M. es Doctor en Ciencias Económicas. Doctor en Ciencias Gerenciales. Magister en Telemática. Magister en Gerencia Empresarial. Ingeniero en Computación y profesor titular de la Universidad Metropolitana del Ecuador, Ecuador.

María Elena Pérez Prieto es Doctora en Gerencia. Maestría en Gerencia de los Recursos Humanos. Licenciada en Administración, Mención Gerencia Industrial. Docente Investigadora del grupo de investigación: Estudios Socioeconómicos, Administrativos y Contables de la Corporación Universitaria del Caribe-CECAR, Colombia.

Marilú Tibisay Acurero Luzardo es Posdoctora en Políticas Públicas y Paz Social. Doctora en Ciencias Gerenciales. Magister Sienciarum en Gerencia Financiera. Ingeniera de Sistemas y Docente investigadora del grupo de investigación: Estudios Socioeconómicos, Administrativos y Contables de la Corporación Universitaria del Caribe-CECAR, Colombia. Categorizada por Colciencias nivel Asociado. 